\title{
Air Quality Index Monitoring System Based on Flow Point of BD
}

\author{
Yuxiang Liu ${ }^{\text {a }}$, Zhengjun Hao, Bing Fan, Rong Tang, Yanan Cao \\ Civil Aviation University of China, Tianjin 300000, China \\ ayxliu_cauc163@.com
}

Keywords: Automatic flow air PM2.5 monitoring, The BD Positioning system, 3G data transmission, tracking robot.

\begin{abstract}
China is annual faced with a large area of bad weather such as fog and haze, which make a serious impact on people's daily lives, traffic congestion and other flight delays. Currently, the monitoring towards air quality are mostly procured through static monitoring station or artificial flow monitoring, whose limitations lie in the incomplete data, heavy workload of the artificial flow monitoring and the poor quality. Even the bad weather may result in serious damage to people. Therefore paper designs a mobile air quality monitoring system. The system uses a robot which has its own air quality monitoring instrument to flow monitoring autonomously; takes the advantage of the BD information systems,3G communication module to locate different sites; adopts the 3G data transmission modules, $\beta$-ray method to monitor the air quality of PM2.5.Besides,the system uses camera to collect relevant environmental information, take the speed and direction of robot into control by video identification guide wire and intelligent PID control algorithm. The air quality monitoring system designed in the paper makes a perfect combination of hardware and software.
\end{abstract}

\section{Introduction}

The Automatic Air PM2.5 ${ }^{[1]}$ Quality inspection can automate the air quality coefficient in bad weather,especially in the haze weather,even far distance.Chinese haze weather is tested by static detection or manual detection, which have very long updated data time,and has caused adverse impact on people's daily life.Therefore,the automatic tracking detection system will become an important means for detecting air quality.The independent research and development of BD satellite system $^{[2-3]}$ has a great prospect, which can provide high accuracy,strong reliability,navigation as well as short message communication ability,particularly the cover of the Asia Pacific region of BD2.

In view of the current efficiency and real-time performance of PM2.5 air quality monitoring,this paper use robots as the carrier to install PM2.5 automatic monitoring module,BD / 3G module is using to gather data and transmit data.The system has practical application value.

\section{The Overall Design of Monitoring System about Flow Air Quality}

\subsection{The Robot's Structure Design to Monitory the Flow of Air Quality}

Considering the robot's moving trajectory in some traffic arteries or complex geographic positions,especially it's operation in the turning and sloping condition,we have designed a three wheeled cylindrical robot ${ }^{[4]}$ whose left and right wheels are driven ones and front wheel is universal active.By the collaborative control of two wheels' rotate speed,we make the robot forward and shift.

To simulate people's awareness, the robot is installed with the omni-directional checking camera, ultrasonic sensors, wind sensor, humidity sensor, infrared sensor and smell sensor etc. The camera simulates the "eye" for tracking; ultrasonic ranging sensor simulates people's vision; the operation of the wind sensor is mainly used for testing the wind series; humidity sensors is used to detect the humidity in the environment; infrared sensor is used to test temperature in different environments by simulating people's feeling; smell sensor simulates robot's "nose" to identify the concentrations of PM2.5 in the air, and can locate the source according to certain methods at the same time ${ }^{[5-6]}$.

To achieve the intelligent navigation of air flow monitoring robot ${ }^{[7]}$, this system adopts a two-level system the structure of parallel robot and control center:the first level is robot automatic actuator 
which controls the respective function modules; the second one is the control center,which can remotely control the robot through $\mathrm{BD}$ and $3 \mathrm{G}$ communication modules.In this two-level system,robot self-control mechanism is used to establish model,on the one hand,it makes the robot run independently,on the other hand,it can make the robot work with the control center simultaneously.The robot system structure is shown in Figure 1.

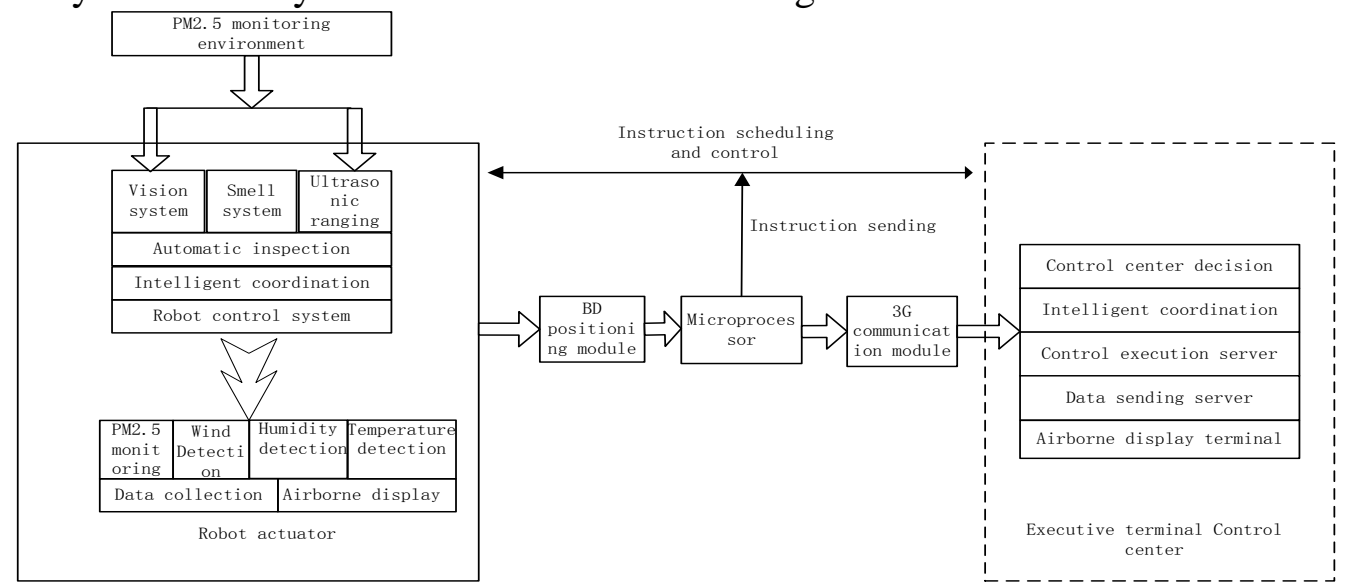

Fig 1.The Overall Structure of PM2.5 Automatic Monitoring

\subsection{Monitoring Robot's Visual Navigation and Tracking Control}

Automatic tracking robot uses cameras to absorb road information,so as to extract "induction" ${ }^{[8]}$ information needed by visual navigation. Video data in this system is YUV format,and resolution size is $1280 * 768$.

There may be makers with different colors in traffic arteries or special environments.This system uses the red "induction",but due to the influences of different lights to the video information acquisition,the YUV color model and RGB color model should be made an integrated test.Data conversion formulas from YUV data to RGB are shown in (1) - (3) :

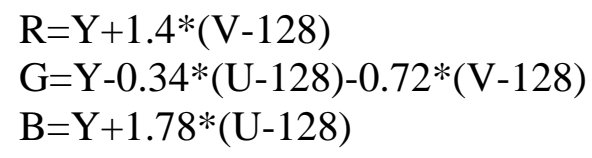

Considering the road condition is poor when the robot in the process of running,red color is leading an error during the acquisition of the data,so this system adopts the introductory section recognition algorithms.According to the different brightness levels,different criteria can be adopted to identify the leading segments.And the leading recognition algorithm can be used to satisfy the requirement of robot automatic tracking operation.

In order to make the robot track "induction" to realize accurate tracking movement,Intelligent PID control method can be used to control robot's left and right directional wheels,and the robot's direction,speed and steering can be controlled.The robot speed control structure diagram is shown in Figure 2.

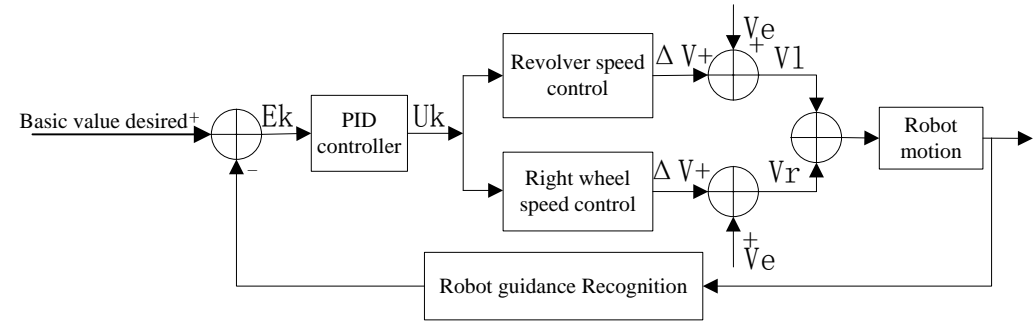

Fig 2.Robot Speed Control Charts

Intelligent PID control method is used in the speed control system,the specific algorithm is shown in (4) - (6) :

$$
\begin{aligned}
& \Delta U_{K}=K_{\mathrm{p}}\left(e_{k}-e_{k-1}\right)+K_{i} * e_{k}+K_{d}\left(e_{k}-2 e_{k-1}+e_{k-2}\right) \\
& U_{K}=U_{K-1}+\Delta U_{K} \\
& U_{K}=U_{K-1}+\Delta U_{K}
\end{aligned}
$$


The $\boldsymbol{e}_{k}$ is the D-value between the normalized abscissa and the abscissa of predetermined in the process of tracking. The $e_{k-1}$ means the last D-value between the normalized abscissa and the abscissa of predetermined in the process of tracking. $K_{\mathrm{p}}, K_{i}, K_{d}$ are the control parameters of the PID controller.

\section{The design of the air quality monitoring}

For many areas of our country adopt static monitoring stations on the air monitoring, so this system adopts the $\beta$ ray absorption ${ }^{[9]}$ and $\mathrm{BD}$ module to monitor the flow of air and sends the latest data to the control center server. At the same time, accurate monitoring data is the basis of the study on PM2.5, while PM2.5 monitoring is complicated (since it is the small part of the various particulate matters floating in the air, which varies differently in the influences of wind, humidity and other factors in different conditions). The precise concentrations of PM2.5 can be monitored by separating PM2.5 out of other particles, at the same time, the isolate weight of the PM2.5 should be measured accurately.

\subsection{The separation of PM2.5}

The PM2.5 monitoring technology at home and abroad is not very mature,so the separating methods used in the PM2.5 is basically the same.Using the special cutter and its special air flow reach the separating effect.The method is when the cutter sucks slipping air,large particles and small particles have different speed so as to achieve the separating effects.

\subsection{The weight measurement of PM2.5}

There are three popular methods to the weight measurement of PM2.5 ${ }^{[10]}$, which are quality method,beta ray absorbing method and micro oscillating balance method.According to liquidity,accuracy and adaptability to the environment of the robot designed in the paper,the method for robot in this paper has the following advantages and disadvantages:quality method needs manual weighing,the process is relatively cumbersome and wasting time.Micro oscillation balance method is using the principle of light scattering,and the higher the concentration of particles in the air,the stronger the scattering of light,by measuring the light scattering degree to calculate the concentration of particulate matter.But because of the same particle structure with different shapes having different degree of light scattering,it is not the same with the mobile environment. And the instrument structure too large to mount on the robot.Beta ray absorbing method is when the beta ray passing the sediment filter, due to the scattering and attenuation, the degree is in the proportional to the weight of PM2.5.The method can be automatically and continuously monitored, and the structure of the device is suitable, so the PM2.5 monitoring of air in this system is based on beta ray absorption method.

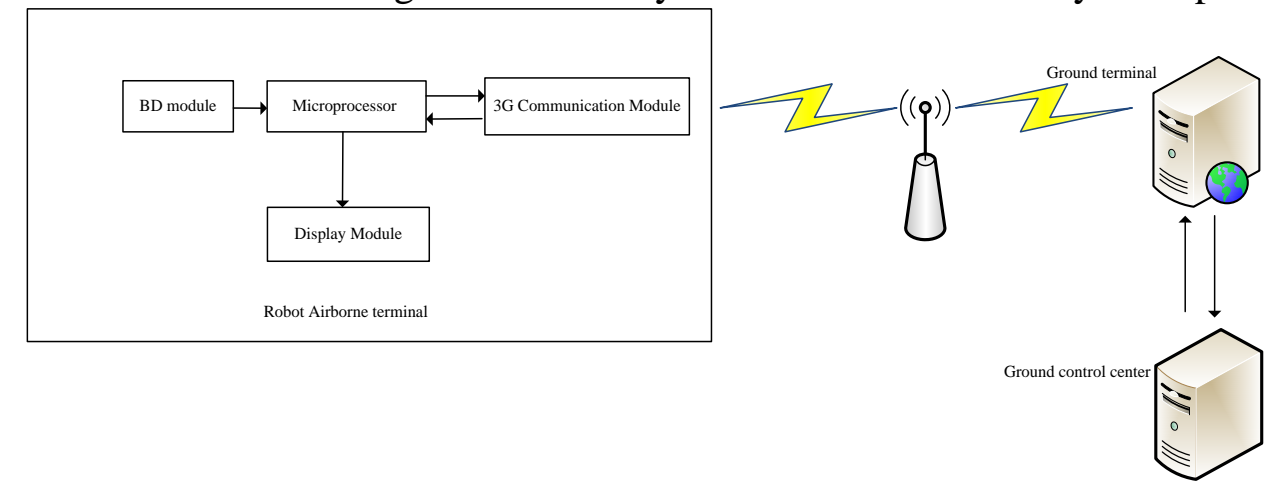

Fig3.BD positioning program structure diagram

\section{BD project design}

\subsection{BD system structure}

The robot localization information is acquired by BD positioning module ${ }^{[11-12]}$, and the required information is transmitted to the server by $3 G$ module.This system uses microprocessor as the core,containing the BD positioning module,airborne display processing module and a server on the 
ground.Using the $3 \mathrm{G}$ data transmission module to establish a data transmission channel to realize the wireless transmission of data between the two places. The system block diagram is shown in Figure 3.

The principle of the $\mathrm{BD}$ location module: using the $\mathrm{BD}$ positioning module robot output the information such as the location. The airborne display system to transmit the air quality index through the 3G module to a server on the ground.The server collect information from all of the robots and promptly sent to display system of various air monitoring station.The ground users can access terminal server through the browser and access to the data of the air quality from robot.

\subsection{BD location algorithm}

BD location system technology is widely used in sea ${ }^{[13]}$, land and air fields and etc.with various locations.It used to adopt the static location measure.Static location measure refers to in the dealing with message of the receiver,the position of point to be located is considered stationary in the ECEF coordinate system.

After obtaining the navigation message of measurement,it is necessary to use these measurements to calculate this fixed position,clock correction and so on.In this paper,it only needs to use the least square method.

When the mathematical model of the observed quantities through navigation message,we can calculate by using the least square method.Assuming that the number of the observed quantities in reality we get is $\mathrm{m}$,so we can get the following observation equation:

$$
\begin{aligned}
& \mathrm{p} 1(x n)=\sqrt{(x n-x s 1)^{2}+(y n-y s 2)^{2}+(z n-z s 3)^{2}}+c^{*} \delta t+n 1 \\
& \mathrm{p} 2(x n)=\sqrt{(x n-x s 2)^{2}+(y n-y s 2)^{2}+(z n-z s 2)^{2}}+c^{*} \delta t+n 2 \\
& \cdots \cdots \\
& \mathrm{p} m(x n)=\sqrt{(x n-x s m)^{2}+(y n-y s m)^{2}+(z n-z s m)^{2}}+c^{*} \delta t+n m
\end{aligned}
$$

In the formula, $x_{u}=\left[x_{u}, y_{u}, z_{u}, \delta t\right]$ is an unknown quantity which is needed to be solved; $n_{i}(i=1 \ldots m)$ is the observation error of each observed quantity. The formula 7 is a non-linear equation,which is needed to be transformed into a linear equation in order to use LS method.The starting from the center coordinates of the ground control center to its BD satellite is always $x_{u o}=\left[x_{u o}, y_{u o}, z_{u o}, \delta t_{o}\right]$, and at this point, the pseudo range equation is spread by using first order Taylor series, obtaining:

$$
\begin{aligned}
& p i\left(x_{u}\right)=p i\left(x_{\text {ио }}\right)+\frac{\partial p i}{\partial x_{u}}\left|x_{\text {ио }}\left(x_{u}-x_{\text {ио }}\right)+\frac{\partial p i}{\partial y_{\text {ио }}}\right| x_{\text {ио }}\left(y_{u}-y_{\text {ио }}\right)+\frac{\partial p i}{\partial z_{\text {ио }}}\left|x_{\text {ио }}\left(z_{u}-z_{\text {ио }}\right)+\frac{\partial p i}{\partial \delta t}\right| x_{\text {ио }}\left(\delta t-\delta t_{o}\right) \\
& H=\left[\mathrm{h}_{i j}\right]=\left(\begin{array}{lll}
\frac{\partial p 1}{\partial x_{u}} \mid x_{\text {ио }} & \frac{\partial p 1}{\partial y_{u}} \mid x_{\text {ио }} & \frac{\partial p 1}{\partial z_{u}}\left|x_{\text {ио }} \frac{\partial p 1}{\partial \delta t}\right| x_{\text {ио }} \\
\frac{\partial p 2}{\partial x_{u}} \mid x_{\text {ио }} & \frac{\partial p 2}{\partial y_{u}}\left|x_{\text {ио }} \frac{\partial p 2}{\partial z_{u}}\right| x_{\text {ио }} \frac{\partial p 2}{\partial \delta t} \mid x_{\text {ио }} \\
\frac{\partial p 3}{\partial x_{u}} \mid x_{\text {ио }} & \frac{\partial p 3}{\partial y_{u}}\left|x_{\text {ио }} \frac{\partial p 3}{\partial z_{u}}\right| x_{\text {ио }} \frac{\partial p 3}{\partial \delta t} \mid x_{\text {ио }}
\end{array}\right)
\end{aligned}
$$$$
\text { arrange the formula (4-10), and record } \delta p=\left[p i\left(x_{u}\right)-p i\left(x_{u o}\right)\right] \wedge T, d x_{u}=\left[x_{u}-x_{u o}\right] \wedge T \text {. }
$$

Bring $\delta p 、 \mathrm{~d} x_{u}, H$ into the formula above and we will get $\delta \mathrm{p}=H * \mathrm{~d} x_{u}+n_{i}$ (9).According to the formula above, we get the Let's solution $\mathrm{d}_{u}=\frac{1}{\left(H^{\wedge} T H\right)}{ }^{*} H^{\wedge} T^{*} \delta_{\mathrm{p}}(10)$

Once getting the correction between initial value and true value after the above formula's first linearization,make use of this correction to update the initial value and then obtain a revised solution of the first time ${ }^{x_{u 1}}=x_{\text {ио }}+\mathrm{d} x_{\text {ио }}$.Regard as the starting point and compute the formula above repeatedly,in this way ,we can constantly obtain new corrected value to update the previous solution.

The above process can be described with a universal formula,for the Nth time of renewal:

$$
\mathrm{d} x_{u k-1}=\frac{1}{\left(H_{k-1} \wedge T H_{k-1}\right)} * H_{k-1} \wedge T^{*} \delta \mathrm{p}_{k-1}
$$

$x_{u k}=x_{u k-1}+\mathrm{dx}_{u k-1}$

In order to distinguish the computed result for each time,subscripts are added to $\mathrm{H}$ and $\delta \mathrm{p}$ in the formula. Because we need to recalculate both of them through the above formula after updating $x_{u k}$ for each time.The end condition of renewal is that when the correction $\mathrm{d} x_{u k}$ meet the predetermined 
threshold value after a calculation,renewal can be stopped and use the consequence $x_{u k}$ as the final calculating result.The end condition can be given by the expression : $d x_{u k}<$ predetermined value.

In conclusion,getting flow of program which is based on the least square method's PTV calculating can be written as the flow chart of the PVT algorithm based on least squares.

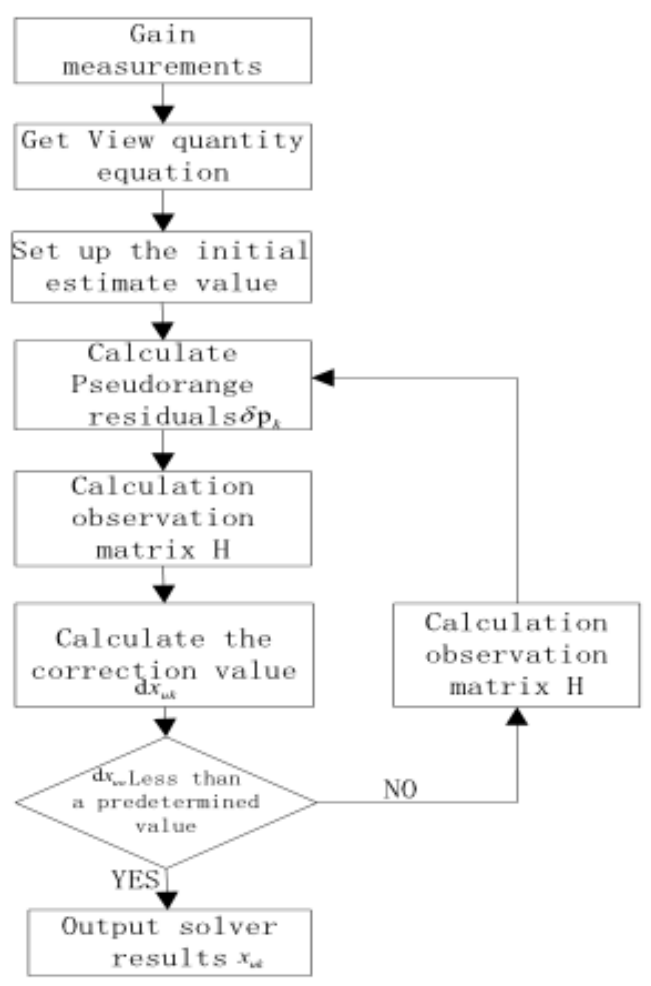

Fig 4.The PVT algorithm based on least squares

\section{3G communication project design}

3G communication module ${ }^{[14]}$ is realized by remote communication, which sends the data collected by the sent to the server. $3 G$ module achieves the communication with the microprocessor by AT instruction, the microprocessor will receive a return value from the GSM module after it sends an AT instruction to the $3 \mathrm{G}$ communication module. After the process of calculation, demodulation and decoding conducted by the sent module, data received by receiving antenna will be output to the microprocessor by USART1 according to the standard of NEMA0183 agreement. At the same time, data detected by the air detecting module will be processed by the microprocessor, then output to the 3G communication module by USARTO, and finally sent to the server by it. As shown in Figure 5.

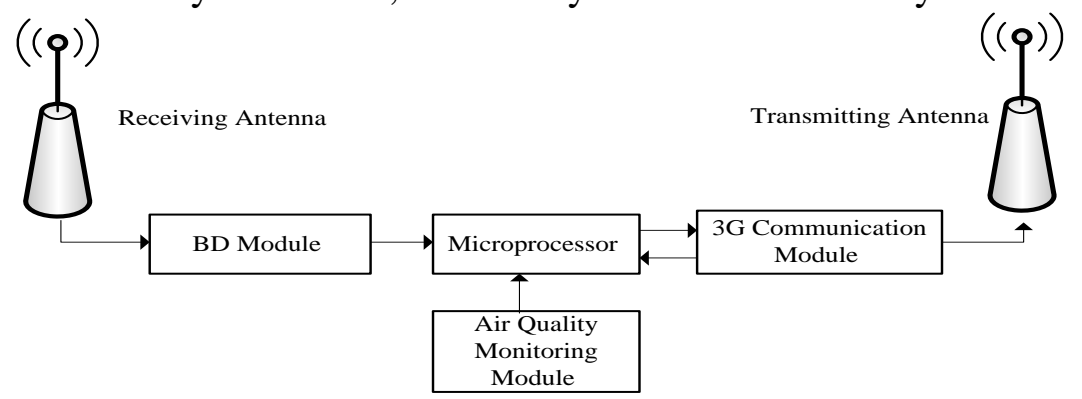

Fig5.3G communication program structure diagram

\section{Airborne display processing module}

In order to display the robot position information and the PM2.5 coefficient information automatically detected on the robot body, also shows other monitoring robots' position and the alarm 
information, airborne display processing module is composed of the following parts: core microprocessor for processing, display module, a $3 \mathrm{G}$ module.

The function of core processing microprocessor is: receiving position data provided by the BD location module, controlling $3 \mathrm{G}$ module to receive position information transmitted from the server on the ground to the other robots and alarm information; dealing with the flow monitoring in the process of the collection of the data processing. Finally, display it on the screen after the integrated processing of its own module. In the course of robot operation, the results of PM2.5 coefficient can be directly displayed to people on the running route, and the shorter period and more direct display to people. Structure as shown in Figure 6.

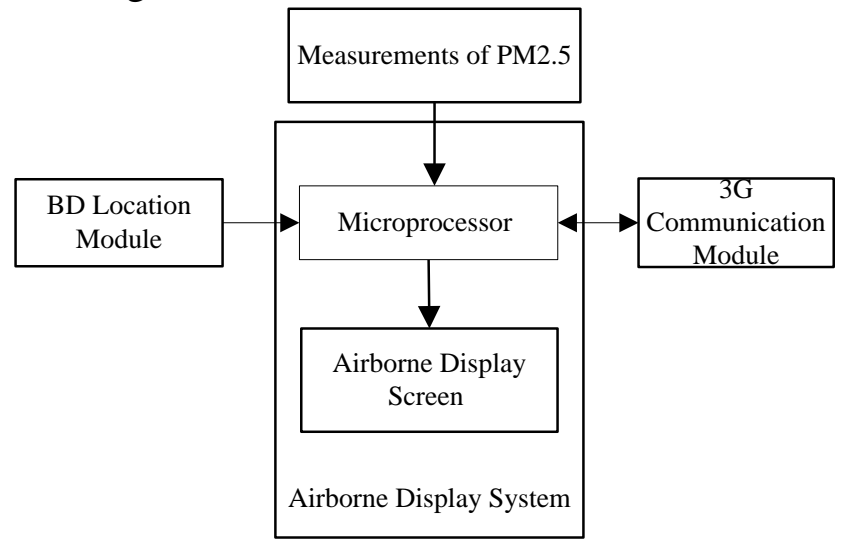

Fig 6. Structure diagram of airborne display module

The main functions of airborne display interface are data display and key operation, and the robot shows the contents of the latitude and longitude of the location information, monitoring PM2.5 coefficient results, wind scale, environmental temperature, and environmental humidity. Key operation including the total start, fault stop, send robot positioning data, monitoring data and other operation buttons.

\section{Conclusion}

The mobile robot automatically monitoring the air PM2.5 coefficient uses BD and other modules of the collaborative work to achieve. Using visual navigation and PID coordinated intelligent control method, to achieve the automatic monitoring, forward, navigation. Using the beta ray absorption device module to monitor PM2.5 coefficient, using BD position system and 3G communication coordination to achieve to locate the robot location information and measure PM2.5 coefficient with efficient supervision and display on the robotic airborne terminal in time, sending the data to the control center server in short period. The system can not only run in unmanned cases, but also can be applied to other occasions, better use of the value of the BD satellite system in China. This system is real-time, and it has improved the monitoring level of PM2.5 coefficient in air, and has a good market prospect.

\section{References}

[1]State Environmental Protection Administration. Indoor Air Monitoring Technology.[S]. 2004.

[2]WANG Hui-nan.Principles and Applications of GPS Navigation, Science Press,Beijing, 2003.

[3]YUAN Jian-ping, LUO Jian-jun,YUE Xiao-kui.Satellite Navigation System: Principles and Applications.[M].China Astronautic Press, Beijing ,2003.

[4]YUAN Ceng-ren, GAO Ming. A Method of Navigation and Collision Avoidance for Mobile Robot in Dynamic Environment, Mar., 2000.

[5]DU Yan-hong, GAO Qing-ji,SUN Cheng-qi,ZENG Hao. Object and Recognition Based on Color and Shape Features.[J].2005,V.25,N.0.

[6]FENG Jian-nong, LIU Ming,WU Jie.Research Advance on Intelligent Navigation for Autonomous Mobile Robot. 
[7]HU Jing-cao.Research on Localization Method for Indoor Autonomous Mobile Robot, Development and Innovation of Machinery and Electric, 2006, N.05.

[8]LI Mao-hai, HONG Bing-rong,CAI Ze-su.A Novel Algorithm for Mobile Robot Global Localization .[J].2006,V34,N03.

[9]PAN Li-na. Research on Sensor Drift in Air Quality Monitoring System. [D].Jun, 2012.6.

[10]LI Ping. Design of Indoor Air Quality Monitoring System Based on ARM. [D].Southwest Jiaotong University, 2010.

[11]CHEN Xiao-long, HUANG Yuan-qing.A Real-time Dynamic Detection System of Formaldehyde in Interior. [J]. Air China Instrumentation, 2007, N.04.

[12]YUAN Jian-ping, FANG Qun.GPS Applied to Positioning and Navigation of Flight Vehicles, Northwestern Polytechnic University Press, Xi'an, 2000.

[13]LIU Jiyu, LI Zhenghang. Principles and Applications of GPS, Mapping Press, Beijing, 1993.

[14]WANG Chunmei, CHEN Jun-jie. Telecommunication Technique Applied to Computer Applications and Modernization of Environmental Monitoring, Feb, 2002. 